ÉGYPTE

monde arabe

\section{Égypte/Monde arabe}

$26 \mid 1996$

Mutations

\title{
Réinventer une " médecine pour tous »
}

Les médecins à la recherche d'une nouvelle crédibilité

\section{Sylvia Chiffoleau}

\section{(2) OpenEdition}

Journals

Édition électronique

URL : https://journals.openedition.org/ema/1472

DOI : $10.4000 /$ ema. 1472

ISSN : 2090-7273

Éditeur

CEDEJ - Centre d'études et de documentation économiques juridiques et sociales

Édition imprimée

Date de publication : 30 juin 1996

Pagination : 81-110

ISSN : 1110-5097

\section{Référence électronique}

Sylvia Chiffoleau, «Réinventer une « médecine pour tous » », Égypte/Monde arabe [En ligne], 26 | 1996, mis en ligne le 08 juillet 2008, consulté le 07 juillet 2022. URL : http://journals.openedition.org/ema/ 1472 ; DOI : https://doi.org/10.4000/ema.1472

Ce document a été généré automatiquement le 7 juillet 2022.

Tous droits réservés 


\section{Réinventer une « médecine pour tous »}

Les médecins à la recherche d'une nouvelle crédibilité

\section{Sylvia Chiffoleau}

1 En Égypte, l'idée du « droit à la santé pour tous » s'est imposée au fur et à mesure que la médecine gagnait en efficacité et que l'État s'appropriait les moyens de son développement. Objet éminemment politique, la santé a donc longtemps constitué un enjeu central des luttes de pouvoir, et l'Égypte contemporaine, successivement confrontée au libéralisme, puis au dirigisme étatique et enfin à la libéralisation économique actuelle, offre un terrain fertile pour observer les variations pouvant exister d'un modèle médical à un autre.

$2 \mathrm{Si}$, aujourd'hui, le rôle de l'État est globalement révisé à la baisse, le principe démocratique de l'accès de tous à la santé ne saurait pour autant être remis en question. Le retrait de l'État s'accompagne donc d'une revalorisation du rôle des professionnels de la santé, et en particulier des médecins, invités à participer plus activement à une réflexion sur la redéfinition des modes d'accès aux soins et de l'offre médicale. Dans ce contexte, on s'attachera ici à examiner comment la remise en cause d'un modèle médical étatique qui, non seulement a déçu les attentes des patients, mais a également dévalorisé le statut professionnel et social des praticiens, donne lieu aujourd'hui à des stratégies professionnelles, individuelles ou collectives qui cherchent à concilier les intérêts catégoriels et la vocation sociale de la médecine.

3 Profession particulièrement prestigieuse, la médecine demeure, partout dans le monde, le symbole de la réussite universitaire et sociale. Le premier des attributs qui la distinguent clairement des autres métiers est le partage entre ses membres d'un titre commun, celui de "docteur ", qui confère aux individus qui le portent une identité singulière et les désigne comme détenteurs d'une compétence exclusive, d'un savoir spécialisé. En retour, ce savoir, qui touche au cœur des angoisses de l'homme, donne à la profession une dimension sociale et humanitaire qui en renforce encore le prestige.

4 À cette position symbolique dans la société, la profession médicale ajoute une position matérielle avantageuse, acquise tout au long du XIX ${ }^{e}$ siècle grâce à une ascension 
sociale continue, généralement dans le cadre de la pratique libérale. Enfin, au cours de la même période, les progrès fulgurants de la discipline octroient aux médecins un pouvoir scientifique, et bientôt politique, qui fait de ceux-ci des acteurs centraux dé la société.

\section{La fin d'une utopie}

\section{De l'âge d'or à la crise}

5 C'est précisément au début du XIX ${ }^{e}$ siècle, au moment où les médecins occidentaux commencent leur ascension scientifique et sociale, que la médecine moderne s'implante en Égypte ${ }^{1}$. Malgré la vocation publique d'abord donnée à la jeune profession autochtone ${ }^{2}$, c'est la représentation idéale du médecin savant, aisé et libéral qui s'enracine sur les bords du Nil. La trajectoire sociale des médecins égyptiens, tout comme celle de leurs homologues occidentaux mais de façon plus lente et hésitante, s'est en effet peu à peu affirmée tout au long du XIX siècle. À l'orée du XX $\mathrm{XX}^{\mathrm{e}}$ siècle, le choix de l'exercice de la médecine correspond, pour une part au moins, à la réalisation d'une ambition sociale. Ainsi, en 1924, un personnage aussi traditionnel qu'Ahmad 'Abd al-Gawwad, dont la personnalité autoritaire domine la trilogie romanesque de Naguib Mahfouz, n'a d'autre ambition que de voir l'un de ses fils devenir médecin, quelque réticence qu'il ait lui-même à faire appel à un praticien en cas de maladie. La conversation qu'il tient avec son fils cadet sur le choix de ses futures études est particulièrement éclairante: il ne s'agit pas de choisir une activité pour son intérêt propre, mais bien pour le prestige social qui lui est attaché et les revenus que l'on peut en attendre ${ }^{3}$. Or il n'y a que la médecine, et avec elle le droit, qui semblent à ses yeux favoriser une telle réalisation.

6 Ahmad 'Abd al-Gawwad ne se trompait guère et l'Égypte de la première moitié du XX siècle a bien assisté à la réussite matérielle de la profession médicale. Le capital économique accumulé par certains à cette époque, dont témoigne encore aujourd'hui leur emprise sur le parc immobilier du Caire et d'Alexandrie, n'a fait que conforter l'opinion généralement répandue que l'exercice de la médecine peut conduire à la fortune. Cette idée est si ancrée dans les représentations qu'elle donne même naissance à une sorte de paradigme résumant un itinéraire professionnel et social accompli qui, encore aujourd'hui, fait se bousculer les candidats à l'entrée des facultés de médecine. Pratiquer la médecine, c'est espérer obtenir les «cinq 'ayn " - du nom de la première lettre des termes désignant les attributs "matériels » auxquels on peut prétendre en tant que médecin : 'arûsa (la mariée), le mariage étant la première étape de l'assise sociale; puis 'arabeyya (la voiture, en arabe dialectal égyptien), attribut de distinction encore relativement rare dans la société égyptienne; ensuite 'iâda (le cabinet de consultation), et 'imâra (immeuble), l'immobilier restant un investissement privilégié de la bourgeoisie égyptienne; enfin 'izba (propriété agricole), symbole le plus évident et le plus considéré d'une fortune assurée.

7 La Révolution de 1952, même si elle modifie profondément les conditions d'exercice de la profession, ne ruine pas pour autant, du moins dans un premier temps, les ambitions sociales attachées à l'exercice de la médecine. Au contraire, celle-ci se pare d'un surcroît de prestige lié à l'engagement au service de la construction nationale et du développement. Dans ce «moment d'enthousiasme $»^{4}$, où l'on s'est pris à rêver d'une 
société libérée de la maladie comme de la pauvreté, la médecine gagne encore en renommée puisqu'elle est désormais réputée pouvoir satisfaire les aspirations sociales de catégories jusque-là exclues de son univers.

Ce caractère prestigieux est d'ailleurs renforcé par le système de sélection universitaire. L'admission dans les différentes facultés égyptiennes est en effet conditionnée par la note obtenue à l'examen de fin d'études secondaires. Depuis sa création en 1961, le ministère de l'Enseignement supérieur décide chaque année du minimum de points requis à cet examen pour prétendre à l'entrée en telle ou telle faculté. Les facultés les plus prestigieuses, celles qui sont censées offrir un meilleur canal de promotion sociale et donc les' plus demandées, sont bien sûr les plus exigeantes. Longtemps les facultés d'ingénierie l'ont disputé à celles de médecine, mais ces dernières se sont finalement imposées à partir de 1967 en n'ouvrant désormais leurs portes qu'aux détenteurs les plus brillants du diplôme de fin d'études secondaires, plaçant ainsi la médecine au sommet des ambitions universitaires ${ }^{5}$.

Même si, aujourd'hui, seules les grandes facultés de médecine du Caire et d'Alexandrie demeurent vraiment au faîte de la pyramide universitaire, certaines facultés médicales de province étant reléguées derrière d'autres disciplines, le prestige de la médecine reste quasi intact malgré le malaise pourtant manifeste qui traverse aujourd'hui la profession. À cette image tenace d'une profession favorisée répond en effet une situation, ou plutôt une série de situations beaucoup moins enviables que ne le laissent supposer les mythes hérités des époques antérieures. En fait, le modèle libéral de pratique en cabinet urbain, qui représente le modèle idéal de l'exercice de la profession, a cédé la place à un fonctionnariat bien moins lucratif, exerçant parfois en milieu rural et qui met quelque peu à mal la précieuse indépendance du praticien.

Le malaise que ressent aujourd'hui une grande partie de la profession médicale égyptienne prend pour certains la forme d'une véritable crise. Les praticiens égyptiens ont sans doute été parmi les premiers à souffrir du chômage dans une profession longtemps épargnée ${ }^{6}$. Pour d'autres, l'insatisfaction est telle qu'ils choisissent, après six ans d'études, de se consacrer finalement à des activités plus lucratives, comme le commerce ou le tourisme, dont l'accès leur est facilité par leur connaissance des langues étrangères. Ce sacrifice du diplôme, consenti pour mieux vivre, est révélateur du déclassement actuel de la profession.

11 Ces symptômes révèlent chez bon nombre de médecins une inadaptation de plus en plus évidente de la représentation de soi à la position réellement occupée. Le désir de réussite sociale, symbolisé par la métaphore des "cinq 'ayn", n'est plus forcément réalisé par la simple obtention du titre ; au contraire, l'acquisition de ces attributs qui définissent matériellement la bourgeoisie devient le fruit d'une lutte où la concurrence est sévère et la morale professionnelle parfois mise à mal. Trahis dans leurs attentes sociales, les médecins se voient aujourd'hui ramenés à un destin beaucoup plus modeste. S'ils cherchent à masquer leur dévalorisation en s'attachant à préserver les marqueurs symboliques qui contribuent à maintenir l'illusion du prestige et de l'aisance, ceci dans un pays où le paraître est primordial, il est aisé de repérer chez certains, dans un costume-cravate élimé ou un véhicule ancien et usagé, les signes d'un niveau de vie bien plus modeste qu'on voudrait le faire croire. À une situation antérieure où la profession montrait un front uni, une homogénéité à la fois sociale et professionnelle, répond aujourd'hui un sentiment d'éclatement, d'atomisation du corps 
médical résultant de la double évolution du système de santé et du système d'enseignement.

\section{La division croissante du travail médical}

12 La profession médicale égyptienne n'est certes pas la seule à subir un tel malaise. Après l'euphorie qui a accompagné la médicalisation globale des sociétés occidentales, au sein desquelles les médecins, grâce notamment à la généralisation des systèmes de protection sociale, se sont vus assurés de la solvabilité de la clientèle ${ }^{7}$, la période actuelle est marquée par le retour de l'incertitude. Confrontée à des problèmes de santé persistants ou à de nouveaux défis, la médecine reste partout hantée par la question de sa propre crédibilité et de celle des hommes qui l'exercent. Ceux-ci sont par ailleurs travaillés par les transformations morphologiques qui affectent leur profession, féminisation et augmentation numérique en particulier, ainsi que par l'évolution scientifique et technique de leur discipline. La crise que traverse aujourd'hui la profession médicale égyptienne participe donc d'un phénomène général qui prend des proportions d'autant plus importantes en Égypte que ce pays connaît, dans les domaines de l'enseignement et de la pratique, des conditions spécifiques qui affectent à leur tour le corps professionnel.

Héritage de la médecine grecque, autant en Occident que dans l'aire musulmane, le diplôme de médecin octroyé à ceux qui ont suivi une formation appropriée est polyvalent et permet en principe d'exercer sa compétence de la façon la plus large, incluant une autorisation d'opérer, donc de pratiquer la chirurgie, évidemment limitée par le bon sens de chacun ${ }^{8}$. Cette capacité théorique d'embrasser l'ensemble du champ médical et d'agir sur tous les organes malades a d'ailleurs largement contribué à asseoir le prestige du médecin.

14 Cependant, l'extraordinaire augmentation des connaissances médicales entrâne un processus de spécialisation qui finit par s'imposer après la seconde guerre mondiale. En Égypte comme ailleurs, les facultés de médecine, devenues alors des institutions solidement établies, offrent des « niches » dans lesquelles certains individus cherchent à promouvoir des spécialités autour desquelles ils organisent une idéologie et un système de rôles professionnels distincts. Cette tendance conduit à une division du travail médical de plus en plus poussée, affectant chacun à des tâches spécifiques et à un domaine exclusif. Dès lors, les spécialités, apparaissent comme un "plus ", une valeur ajoutée par rapport à la connaissance médicale supposée maitrisée par l'ensemble des praticiens.

15 Ces conditions nouvelles entraînent pour le médecin la perte d'une partie de ses prérogatives: il ne peut plus être considéré comme omniscient dans son domaine, malgré l'étendue théorique du champ d'action accordé par son diplôme. La division du travail médical a ainsi quelque peu érodé la fierté professionnelle du médecin qui doit désormais faire appel à d'autres, déléguer une partie de son travail à des individus perçus comme plus performants. Le praticien-artisan doit céder la place à un travail d'équipe où interviennent des spécialistes et des techniciens nouveaux, formés à la manipulation de technologies de pointe devenues, dans certains cas, indispensables au diagnostic.

16 La logique de l'apparition des spécialités, qui distingue certaines zones où s'installe un savoir ou un savoir-faire particulier et performant, conduit en retour à l'invention de la 
notion de médecine générale. Celle-ci, en effet, est devenue « ce qui reste » une fois que le système des spécialités s'est mis en place ${ }^{9}$, une sorte de résidu moins prestigieux offrant finalement à ceux qui l'exercent des conditions sociales et professionnelles dévalorisées.

17 À cette hiérarchisation de la profession par la spécialisation s'ajoute une grande différenciation technique au sein même du système de santé qui prend, dans un pays comme l'Égypte, une visibilité accrue. Après avoir été longtemps sous-équipée dans le domaine sanitaire, l'Égypte opte finalement, au début des années soixante, pour un système public de santé donnant la priorité à la couverture sanitaire des zones rurales. Cependant, malgré le souci affirmé d'offrir un système médical de base à l'ensemble de la population, ce sont les services sophistiqués et prestigieux qui demeurent au cœur des préoccupations des professionnels comme des politiques. Pour les premiers, ils constituent un espace où peut s'épanouir le talent, un symbole de la modernité et un gage de leur compétence. Pour les seconds, ils expriment la volonté de rester dans la course des sciences et de la modernisation. Gamal Abdel Nasser avait déjà tenté de mettre en place une politique scientifique et de développer un secteur médical de pointe; son successeur a renforcé cette tendance en ouvrant largement les portes au transfert des technologies.

Ainsi l'Égypte se trouve désormais être parmi les pays arabes les plus grands consommateurs de technologie médicale ${ }^{10}$. Le système de santé égyptien comprend donc, à l'autre extrême d'une ligne qui part du réseau rural de soins, une série d'hôpitaux à haute technologie, équipés d'appareillages sophistiqués exigeant des compétences techniques et des qualifications de plus en plus spécialisées. Cette vitrine médicale moderne absorbe une minorité de praticiens, les mieux formés et les plus performants qui, à leur tour, réclament les équipements les plus récents, entraînant le système de santé dans une spirale coûteuse de consommation technologique. À l'opposé, la base de la pyramide sanitaire, dépourvue de moyens et de matériel, emploie une majorité de praticiens, essentiellement des généralistes, dans des conditions d'autant moins satisfaisantes que leur nombre dépasse de beaucoup les structures disponibles.

\section{Une production de masse}

19 Depuis la Révolution de 1952, le système universitaire égyptien s'est développé de façon considérable, avec pour mission de former une élite nationale à même de prendre en charge un programme de transformation de la société conduit par l'État, et d'assurer au plus grand nombre un canal légitime et effectif de promotion sociale. À partir de 1962, l'enseignement supérieur devient gratuit et, deux ans plus tard, une loi garantit l'embauche de tous les diplômés dans les structures publiques, renforçant ainsi l'attrait exercé désormais par la fonction publique sur les nouvelles catégories éduquées à la recherche de stabilité.

Ce programme suppose l'extension des structures universitaires, limitées en 1952 à trois établissements. L'université d'Assiout voit finalement le jour en 1957, suivie d'une dizaine d'autres dans la plupart des grandes villes du pays. Ainsi, de trois en 1952, le nombre des facultés de médecine est passé aujourd'hui à quatorze. Dès le début des années soixante, le nombre des étudiants en médecine fait un bond considérable. Les trois facultés comptent un total de 3125 étudiants en 1959-60 ; ils sont 13 379, il est vrai 
dans quatre facultés, en 1963-64. Cette augmentation très importante correspond bien sûr à un élargissement de la base du recrutement universitaire. Les enfants des classes moyennes, des commerçants ou des petits propriétaires ruraux, considérés comme les principaux soutiens du nouveau régime, ont désormais accès à l'université, censée leur offrir un canal légitime de promotion sociale. La profession médicale connaît alors une véritable explosion, passant de 17000 médecins en 1963 à 32000 en 1977 et à 100500 aujourd'hui.

21 Une telle pléthore, qui dépasse largement les capacités d'accueil du système universitaire, s'accompagne d'une dégradation du niveau d'enseignement, sensible depuis le début des années soixante-dix ${ }^{11}$, due à la surcharge des amphithéâtres et au manque d'équipements dans les facultés. Les classes contiennent jusqu'à trois fois leur capacité : s'il est inconfortable de suivre un cours magistral dans ces conditions, l'assimilation des cours cliniques y devient quasi impossible. On compte fréquemment cinquante étudiants autour d'un microscope ou d'un cadavre, ce qui rend difficile toute observation sérieuse. En outre, les hôpitaux universitaires, où les étudiants sont censés s'initier à la pratique, sont en nombre nettement insuffisant, au point que les patients n'hésitent pas à réclamer quelque argent pour se laisser examiner.

Pour réussir malgré tout aux examens, les étudiants doivent donc avoir recours au système des leçons particulières, devenues une véritable institution dans la mesure où elles représentent une source importante de revenus pour des enseignants rémunérés au tarif dérisoire de la fonction publique. Ces derniers sont par ailleurs beaucoup plus intéressés par leur pratique privée que par leur poste d'enseignement, et les étudiants déplorent la distance du corps enseignant à leur égard, quand ils n'ont pas à se plaindre de son mépris.

Un tel système et ses dysfonctionnements tendent finalement à créer une sorte de formation à deux vitesses, où les étudiants issus de milieux favorisés peuvent combler leurs lacunes grâce aux cours particuliers, à la lecture d'ouvrages onéreux et à des stages effectués à l'étranger. Quant aux autres, ils doivent se contenter d'une formation très imparfaite et de connaissances souvent floues qui en font des praticiens peu sûrs d'eux-mêmes et sujets aux erreurs de diagnostic. Le déséquilibre est également frappant entre les grandes universités du Caire et d'Alexandrie, qui forment des médecins souvent performants, et les universités de province, particulièrement les dernières nées, dépourvues de moyens financiers et dont le corps enseignant se distingue surtout par son absentéisme. Les diplômés de ces facultés de médecine ne reçoivent qu'un apprentissage limité qui les assimile plus à des "officiers de santé " qu'à de véritables médecins. Cette formation médiocre et leurs origines sociales modestes les destinent dans leur majorité à un emploi dans la fonction publique. Or le modèle médical offert par la Révolution de 1952 n'a pas tenu ses promesses et, à un niveau scientifique et une position sociale détériorés, les médecins ajoutent un fort sentiment d'insatisfaction dans l'exercice de leur profession.

\section{L'héritage ingérable du nassérisme}

\section{Le droit à la santé pour tous}

Avec plus ou moins de poids et d'importance selon les époques, un secteur public de santé a toujours existé en Égypte, cohabitant avec un secteur privé souvent plus visible et toujours plus prestigieux. L'idéologie dont est porteuse la Révolution de 1952 donne 
un relief nouveau au principe de la prise en charge étatique de la santé et du traitement des maladies. Le nouvel État, riche d'espoirs et d'ambitions, se modèle sur un dirigisme économique destiné à satisfaire l'ensemble des besoins de la population, incluant des services tels que la santé ou l'éducation. Les articles 16 et 17 de la Constitution égyptienne attribuent à l'État la mission de fournir aux citoyens les services culturels, sociaux et médicaux nécessaires, principe réaffirmé par la Charte nationale de 1962 qui accompagne le choix d'une option « socialiste " pour le développement de l'Égypte ${ }^{12}$.

Cette notion de «droit à la santé » pour l'ensemble de la société suppose une extension des obligations de l'État: c'est en effet à lui, et à lui seul, que revient le devoir de fournir les structures de soins correspondant à une médicalisation globale et équitable. $\mathrm{Du}$ même coup, s'opère une sorte de renversement des rôles entre État et corps médical. Alors que l'État gérait ce que les médecins proposaient, ce sont désormais les médecins qui sont chargés de suivre les priorités imposées par l'État. En effet, les membres du corps médical sont promus au rang d'acteurs privilégiés et centraux d'un dessein de modernisation national et invités à rompre avec une tradition professionnelle trop nettement individualiste. La vocation d'experts qu'ils se sont assignée doit être mise au service de la société et des objectifs dictés par l'État.

Cette vocation nouvelle donnée aux médecins, leur mobilisation par un État qui entend faire d'eux les instruments de sa politique sociale, entrent rapidement en conflit avec les intérêts d'une profession qui compte bien, de son côté, perpétuer son statut social et les avantages matériels liés à la pratique privée. Très rapidement, la profession médicale se heurte au nouveau régime et c'est le syndicat des médecins, fondé en 1940, qui devient le lieu de l'affrontement entre les deux parties. Dès 1953, le régime issu de la Révolution fonde un nouveau journal ${ }^{13}$, al-Atibbâ', qui devient l'organe officiel du syndicat des médecins. En réaction contre la mainmise, sur ce dernier, d'une élite médicale jugée représentative de la bourgeoisie de l'ancien régime, ce journal se veut l'organe d'expression des médecins de province, pour la plupart généralistes et intégrés au système public de santé, même s'ils ont souvent, par ailleurs, une pratique privée. Cette réhabilitation des médecins de base aux avant-postes de la conquête médicale vise à les mobiliser en faveur du régime et contre l'élite médicale étroite qui monopolise une grande partie des structures de soins privées. Le rédacteur en chef de cette publication demeurera longtemps le Dr al-Nabawî al-Muhandis, l'une des figures de proue du syndicat revisité par les nassériens, qui deviendra plus tard ministre de la Santé et sera notamment l'artisan de l'implantation du réseau rural de soins au début des années soixante.

Les syndicats professionnels, qui comptent parmi les rares institutions à avoir survécu à l'ancien régime, sont en effet promptement investis par les partisans de la Révolution. La pression de ceux-ci sur le syndicat s'accentue, au début des années soixante, avec le virage socialiste du régime et la création de l'Union socialiste arabe. L'enjeu du conflit, au sein du syndicat, entre État et profession médicale est alors dramatisé dans la mesure où le premier veut imposer à la seconde un système de santé entièrement socialisé, dans lequel les médecins perdraient toute possibilité de pratique privée. Cependant, les médecins se montrent assez forts ou déterminés pour parvenir à un compromis: ils acceptent un contrôle étatique de la pratique privée ainsi que certaines obligations, sans être pour autant obligés de participer à un programme national de santé. Il est vrai qu'en la circonstance, le nombre encore réduit de médecins dans le pays joue en leur faveur: il ne sont que 17000 en 1963, au moment où l'État 
cherche précisément à étendre largement les services de santé. Cette capacité de résistance doit sans doute être également mise sur le compte de l'image du médecin, que le pouvoir nassérien n'est pas parvenu à modifier entièrement, et du prestige dont il continue à bénéficier. Cependant, bon gré mal gré, et bien qu'ils aient réussi à conserver certaines prérogatives, les médecins sont invités dans leur majorité à rejoindre un système général de santé publique, gratuit pour l'usager. La plupart de ces postes dans la fonction publique, notamment en milieu rural, sont désormais incompatibles avec une pratique privée.

Si l'élite médicale voit dans ce système la fin de ses privilèges, pour beaucoup, le modèle sur lequel il repose offre au contraire l'espoir de réaliser enfin une couverture sanitaire complète et efficace de la société égyptienne. Dans le contexte de la naissance de l'Etat national, la santé devient l'un des symboles de la modernisation et de la victoire sur le sous-développement. Les espérances placées dans les progrès médicaux sont d'ailleurs d'autant plus grandes qu'on ne soupçonne pas de limites à la discipline. Dans ce contexte, qui culmine dans les années soixante avec l'implantation du réseau rural de santé - l'un des plus denses des pays en développement-, apparait la figure du "médecin-militant» attentif à sa mission sociale autant qu'à son devenir professionnel. Ainsi Youssef Idris, médecin avant d'être écrivain, commence-t-il sa carrière médicale en occupant les modestes fonctions de responsable du Bureau de santé $^{14} d^{\prime} u n$ quartier très populaire du Caire. Cette expérience lui inspire une nouvelle où il décrit avec émotion la tâche quotidienne du médecin de santé publique, tâche routinière mais qui prend sous sa plume une dimension profondément humanitaire ${ }^{15}$.

\section{La faillite du système}

29 À la fin des années quatre-vingt, le jeune médecin attaché au poste occupé autrefois par Youssef Idris est bien loin de manifester le même enthousiasme pour sa mission. Les locaux des bureaux de santé sont vétustes et d'une propreté plus que douteuse; le matériel aussi indispensable que les seringues ou le coton est nettement insuffisant et le personnel paramédical, incompétent et absentéiste. Cette situation, peu faite pour satisfaire les attentes sociales et professionnelles du praticien, est surtout révélatrice de l'échec d'un modèle qui a trouvé ses limites dans son utopie même. En effet, dans un système reposant sur le service public, l'ensemble des revenus de l'État est redistribué dans les différents secteurs afin de faire fonctionner une machine économique qui reste en grande partie non productive, dans la mesure où les services ainsi offerts sont gratuits pour l'usager. Bien entendu, un tel système ne peut se satisfaire que d'une conjoncture économique favorable. Après l'euphorie des années soixante où l'on croyait l'État-providence capable de tout offrir, le financement des grandes machines économiques reposant sur le " tout-public » s'est révélé de plus en plus difficile. Malgré les élans de rhétorique faisant de la santé une priorité de l'État, la part de ce secteur dans le budget est réduite à la portion congrue. Ainsi, de 1936 à 1965, elle oscille entre $3,7 \%$ et $5 \%$, chiffre record de 1965 qui a permis de réaliser une avancée importante dans la couverture sanitaire des zones rurales. En 1970, cette part est réduite à 1,6\% du budget pour chuter encore - à $1,1 \%$ - en $1975^{16}$. Jusqu'en 1982, les Plans successifs concernant la santé ne sont publiés qu'irrégulièrement et avec retard, et les budgets sont parfois révisés à la baisse.

Les années soixante-dix ont été particulièrement peu propices au secteur de santé. Alors que la décennie précédente s'était attachée à multiplier les équipements de soins 
dans les zones rurales, cette période, qui a pourtant connu une véritable explosion démographique dans les zones urbaines, n'a pas été en mesure de s'adapter et de suivre cette évolution rapide en ce qui concerne la couverture sanitaire de la population. Ainsi, les quartiers urbains périphériques et, en particulier, les zones d'habitat spontané se trouvent largement sous-équipés. Au début des années quatre-vingt, il y avait une unité de soin pour 3000 familles en zone rurale, alors que les zones urbaines n'en avaient qu'une pour 16000 familles $^{17}$.

Depuis le début des années quatre-vingt, la santé a retrouvé une certaine place dans les préoccupations de l'État, bien que la politique s'infléchisse dans un sens plus libéral. La part du budget de l'État octroyé au domaine de la santé est remontée aux alentours de 2 à $2,5 \%$ depuis $1985^{18}$, mais ce montant, encore fort éloigné des $7 \%$ considérés comme adéquats par les organismes internationaux, reste tout à fait insuffisant pour faire fonctionner de façon décente un système de santé de cette envergure.

Dans ces conditions, le réel effort de mise en place de structures médicales est considérablement entravé par l'incapacité dans laquelle se trouve l'État d'assurer la maintenance et la reproduction de ce système. Le primat du fonctionnariat ne fait qu'aggraver les contraintes financières et la répartition du budget entre les différents postes révèle nettement les difficultés auxquelles la gestion du secteur de la santé doit faire face. Plus des deux tiers du budget de la santé sont ainsi consacrés au paiement des salaires, $20 \%$ à la maintenance des équipements et aux dépenses de médicaments (ces derniers représentant à eux seuls les deux tiers de la somme), les investissements nouveaux ne bénéficiant que de $12 \%$ de l'ensemble ${ }^{19}$. Les équipements construits dans les années cinquante-soixante, tout particulièrement à la base du système de santé intégré, sont donc désormais obsolètes, le matériel l'est également et les ruptures de stock de médicaments pratiquement constantes. Certains bâtiments sont si vétustes et dépouillés de tout qu'il semble difficile d'y envisager quelque acte médical que ce soit. Par manque de motivation et de moyens, l'entretien quotidien des équipements est négligé jusqu'à provoquer parfois la fermeture des locaux pour des raisons d'hygiène, ou encore du fait de la désertion des personnels médicaux censés y exercer.

\section{Les médecins-fonctionnaires : un groupe désabusé}

Les praticiens engagés dans la pratique publique, qui constituent encore la majorité du corps médical ${ }^{20}$, notamment ceux qui occupent les échelons inférieurs du système, sont donc en permanence confrontés à la pénurie. Dans l'exercice quotidien de sa profession, le médecin est amené à jongler avec les contraintes du système de façon à se rapprocher au plus près de la réalisation de la tâche qui lui est dévolue. Ayant pour vocation de soigner, ce qui revient le plus souvent à faire don de médicaments, il se voit pourtant dans l'impossibilité de répondre à l'attente de ses malades, ce qui remet en cause à ses yeux les fondements même de son travail. Cette pénurie quasi permanente donne lieu à des attitudes de renoncement agressif où le médecin, d'un ton souvent peu gracieux, renvoie le patient à la pharmacie privée où il obtiendra, moyennant argent, un médicament qu'il aurait pu avoir gratuitement dans l'unité de santé. Ou, au contraire, elle aboutit à un dévouement et des efforts qui, sans être vraiment rares, ne sont cependant pas la règle.

En plus des problèmes rencontrés dans l'exercice même de sa fonction, le médecin doit également faire face à ses propres difficultés financières. En effet, les salaires offerts aux praticiens employés dans le service public de santé sont équivalents à ceux de 
n'importe quel fonctionnaire égyptien. Ainsi, un médecin débutant gagne, en 1995, 80 à $90 \mathrm{Ee}^{21}$ par mois (soit environ $130 \mathrm{~F}$ ), somme tout à fait insuffisante pour vivre de façon décente et, a fortiori, pour maintenir le niveau de vie traditionnellement associé à l'exercice de la profession médicale. La grille financière d'avancement, qui n'a pas été modifiée depuis 1979 malgré une forte inflation, évolue par ailleurs fort lentement; cette situation se prolonge donc sans amélioration significative pendant toute la durée consacrée à la pratique en secteur public.

La médiocrité des conditions offertes par le secteur public de santé, tant d'un point de vue professionnel que social, conduit les médecins à condamner ou rejeter de façon plus ou moins radicale le système dans lequel ils sont engagés. Pour certains, ces conditions sont si inacceptables qu'il n'est pas question d'accorder le moindre crédit à un système public qui a fait les preuves de ses insuffisances. Ceux-là ont une représentation très manichéenne du domaine médical. D'un côté, il y aurait un service public de santé "primitif et fantoche" où rien n'est possible, où l'exercice de la médecine, déshumanisé et routinisé, perd totalement son sens et où le praticien, dépourvu de tout, est incapable de réaliser sa tâche. D'un autre côté, il y aurait un lieu où s'exerce une médecine " authentique » ou "normale ", c'est-à-dire efficiente, et qui serait le secteur privé. Contre une certaine vision qui fait de ce dernier une activité essentiellement commerciale, ceux qui le défendent y voient au contraire la seule formule qui permet d'exercer une médecine humaine et attentive, en garantissant la disponibilité du médecin grâce à un salaire décent et la motivation du patient par le paiement d'honoraires.

Pour d'autres, la condamnation est moins ferme dans la mesure où la gratuité, ou du moins, le très faible coût pour l'usager des structures du ministère de la Santé, leur semble indispensable dans un pays à bas revenus et dont la frange la plus défavorisée de la population est moins que jamais en mesure, en raison de la crise économique, d'assumer les frais de santé. Le secteur public n'est donc pas vraiment attaqué de front, dans son principe, mais c'est l'État, ordonnateur du système, qui est mis en cause, accusé de mauvaise gestion, d'avarice ou d'incohérence dans sa politique de santé. Dans son ensemble, la profession médicale se montre donc très critique envers le modèle proposé autrefois par le pouvoir nassérien. Les plus virulents se comptent sans doute parmi la jeune génération, la plus touchée par la dégradation des conditions de vie et d'exercice, et pour laquelle les mots d'ordre de l'idéologie socialiste n'ont plus guère de sens.

\section{Un phénomène de génération}

37 La tendance croissante à la spécialisation du corps médical, les progrès technologiques constants au sein de l'hôpital ainsi que la surproduction universitaire par rapport à l'infrastructure sanitaire disponible conduisent finalement à une bipolarisation de la profession médicale, opposant un establishment qui jouit d'une situation prédominante à une large fraction des étudiants et des praticiens de base qui rencontrent de grandes difficultés à s'imposer, aussi bien au sein de l'Université que dans le champ de la pratique. Malgré les tentatives menées par le pouvoir nassérien pour briser la pratique privée, l'élite de la profession issue de la période libérale est parvenue à maintenir ses privilèges et à sauver une grande partie de ses biens. C'est bien sûr cette élite qui occupe la plupart des postes prestigieux du secteur de pointe ainsi que les positions académiques. La reproduction au sein de cette catégorie étant, par ailleurs, 
particulièrement importante, le transfert par héritage des cliniques privées et de la clientèle solvable clôture de façon assez hermétique ce groupe privilégié qui ne représenterait que $8 \%$ environ de l'ensemble de la profession ${ }^{22}$.

À l'autre pôle du corps médical, on rencontre la cohorte des médecins qui ont bénéficié de l'accès à une université recrutant désormais sur une base sociale élargie. À l'exception des individus issus du milieu médical même ou des catégories sociales les plus favorisées, ces nouveaux praticiens se heurtent à leur sortie de la faculté à l'insuffisance des structures de santé et à leur propre incapacité à s'installer dès leurs débuts dans une pratique privée stable, ce qui diffère pour de longues années la réussite sociale et professionnelle à laquelle ils aspiraient.

La bipolarisation de la profession recoupe donc en grande partie un phénomène de génération. En effet, la majorité des médecins actuellement sur le marché médical égyptien ont été formés au cours des vingt dernières années et ils sont confrontés, de par leur nombre et l'état des structures de soins, à des difficultés spécifiques et souvent inédites dans le pays. C'est dans le courant de la décennie soixante-dix que le problème est apparu comme tel aux observateurs et l'on parle depuis lors, autant dans la presse qu'au sein même de la profession, de « la crise des jeunes médecins » pour désigner les obstacles actuellement rencontrés, au cours de leur itinéraire professionnel, par la nouvelle génération.

L'apparition de cette notion de crise correspond au constat, de plus en plus évident dans la société égyptienne, que l'identité nominale, le titre de "docteur », dissimule en fait une disparité bien réelle et que les " grands médecins » (al-atibbâ' al-kubâr), c'est-àdire les praticiens établis solidement en pratique privée ou hospitalière, et non pas seulement les professeurs de médecine, ont bien peu de traits communs avec les "jeunes médecins » (al-atibbâ'al-shubbân). L'insuffisance des infrastructures sanitaires empêche ces derniers de jouir des profits matériels et symboliques attendus de l'exercice de la médecine. Par ailleurs, l'expansion universitaire, en banalisant dans une certaine mesure l'accès à la profession, entraîne une diminution de la valeur attachée au titre. Cette catégorie des jeunes médecins se trouve donc dans une position inconfortable entre ce qui devrait être - la réussite et l'aisance - et ce qui est en réalité - une pratique professionnelle décevante et une position sociale instable. Elle éprouve désormais bien des difficultés à se reconnaître dans une identité collective qui fait du médecin un praticien libéral, un homme qui a réussi, alors que sa propre situation reflète un déclassement aussi bien culturel que social.

41 Les trois-quarts des médecins actuellement inscrits au tableau de l'Ordre ayant moins de quarante ans ${ }^{23}$, ce qui en fait une profession exceptionnellement jeune, c'est en réalité la majorité de la profession qui est touchée par l'ensemble de ces problèmes. Un sondage effectué auprès de ses lecteurs ${ }^{24}$ par le journal Al-Atibbâ' destiné à élaborer une réforme en vue d'améliorer les revenus de la profession, permet de préciser les caractéristiques du «jeune médecin ». La quasi-totalité des individus qui ont répondu au questionnaire ayant moins de quarante ans, l'un des chiffres les plus remarquables révélés par ce sondage est le taux de célibat, qui s'élève à $43 \%$ chez une population pourtant en âge de se marier. En Egypte, la pression sociale s'accentue sur le rituel du mariage et sur la cérémonie à laquelle il donne lieu: les contraintes matérielles des parties y sont étroitement précisées. Pour prétendre au mariage, un "aspirant bourgeois » doit pouvoir offrir à la jeune épousée un appartement meublé, des bijoux et, d'une manière générale, une existence décente. La pression est d'autant plus forte 
dans les classes dites "moyennes» que le mariage y apporte, plus qu'en d'autres secteurs sociaux, le témoignage, la preuve de l'aisance matérielle. Pour les médecins, supposés appartenir à cette catégorie, le problème est délicat dans la mesure où, contrairement à l'attente générale, ils sont bien souvent dans l'incapacité de rassembler à l'âge courant du mariage, soit $25-30$ ans, la somme nécessaire à la formation d'un foyer. En fait, l'investissement financier que l'on doit consentir aujourd'hui pour se marier diffère pour longtemps la réalisation du premier objectif contenu dans le paradigme des "cinq 'ayn». La voiture reste bien souvent aussi un objet inaccessible; quant à l'immeuble et à la propriété agricole, ils relèvent du pur fantasme.

Issus de milieux familiaux à revenus moyens, qui ne leur permettent pas d'accéder dès la sortie de la faculté à une pratique privée, la majorité des médecins actuellement formés doit se contenter des conditions peu satisfaisantes offertes par les structures publiques de soins. Ou bien il leur faut se mobiliser pour inventer des formules alternatives qui offriraient les mêmes garanties d'universalité que la médecine de santé publique, mais dans une formulation plus propre à satisfaire leurs attentes sociales, projet qui trouve un écho favorable dans le contexte actuel de libéralisation.

\section{Tendances de la privatisation dans le domaine médical}

\section{Une diminution des interventions de l'État}

En entrant dans l'ère du pétrole arabe, l'Égypte est rapidement conduite à réviser les politiques - et le substrat idéologique sur lequel elles reposaient - qu'elle avait suivies depuis bientôt trois décennies. La politique d'ouverture économique (infitâh) entamée à partir de la moitié des années soixante-dix se propose de jouer la carte du libéralisme économique. Dans le bilan de la période précédente, force a été, en effet, de constater que les modèles proposés n'ont pas réalisé toutes leurs promesses et que la démocratisation a toujours été remise à plus tard. Du coup, en escomptant ainsi un surcroît de légitimité, le pouvoir actuel se désigne comme seul capable de mener à terme le processus démocratique et d'arbitrer la privatisation des différents secteurs sociaux en respectant les impératifs de la justice sociale. En effet, l'État renonce à tout assumer mais veut continuer à se porter garant d'une redistribution équitable des ressources collectives. Ainsi, dans le domaine de la santé, particulièrement sensible en raison de son implication sociale, le désengagement de l'État s'est accompagné d'une tentative d'amélioration des activités du secteur public, destiné en principe aux fractions les plus défavorisées de la population. Le principe nouveau repose en effet sur l'idée que l'État ne peut plus tout financer et que ceux qui peuvent payer doivent contribuer aux dépenses. La notion de gratuité a fait long feu et, désormais, l'État limite son intervention sociale au bénéfice des plus démunis.

\section{L'enfance : un groupe cible}

Les enfants, particulièrement vulnérables dans un contexte de paupérisation, sont les premiers à retenir l'attention des responsables des politiques de santé. Malgré l'existence d'un système de santé ancien et implanté sur l'ensemble du territoire, l'Égypte est encore victime, à la fin des années quatre-vingt, d'un taux de mortalité 
infantile qui compte parmi les plus élevés du monde. Ce taux, estimé par l'Unicef à 129 pour 1000 en $1989^{25}$, chiffre comparable à celui qui caractérise la situation de nombreux pays africains, est bientôt apparu intolérable. Le resserrement des politiques de santé autour de groupes cibles tels que celui de l'enfance s'est ajusté par ailleurs à un courant encouragé par les instances internationales. Alors qu'au cours de l'année 1988, un groupe de travail des Nations Unies discute un projet de Convention des droits de l'enfant ${ }^{26}$, le président Moubarak inaugure en Égypte un Conseil national pour l'enfance et la maternité, destiné à coordonner et à promouvoir les initiatives en matière d'aide à l'enfance, notamment en ce qui concerne les problèmes de santé. À cette occasion, le président égyptien proclame « une décennie pour le développement de l'enfant égyptien, 1989-1999», qui doit aboutir à la réalisation de neuf objectifs prioritaires, dont cinq visent l'amélioration des conditions de santé.

À mi-parcours, le bilan de cette décennie consacrée à l'enfance apparaît cependant assez limité. La principale réussite tient dans l'augmentation importante du taux de vaccination pour six maladies particulièrement meurtrières (poliomyélite, rougeole, tétanos néonatal, diphtérie, tuberculose et coqueluche), qui a entraîné une chute spectaculaire du taux de mortalité infantile, désormais stabilisé autour de 60 pour $1000^{27}$. Cependant, ces résultats positifs doivent beaucoup au fait que le programme élargi de vaccinations, lancé par l'Unicef et l'OMS en 1974, mais surtout développé à partir de 1985, qui avait pour objectif ambitieux de vacciner tous les enfants du monde en 1990, a reçu une aide internationale conséquente. Une initiative comme celle de la décennie pour le développement de l'enfant égyptien apparaît surtout comme une tentative de l'État de préserver une légitimité menacée, afin de contrebalancer son renoncement progressif à ses fonctions distributives et idéologiques.

\section{Les limites d'un système d'assurance maladie}

L'autre orientation principale des politiques de santé, qui vise à compenser les effets négatifs de l'infitâh, concerne le développement de l'assurance maladie. Mis en place en 1964 puis révisé en 1975, le système d'assurance maladie ne concerne que les employés de l'industrie, notamment publique, et les fonctionnaires du secteur bureaucratique gouvernemental, invités à participer à l'effort de financement par un système de cotisations sur salaire ${ }^{28}$.

Dans un contexte de libéralisation, l'assurance maladie apparait comme la formule de moyen terme la plus fiable entre secteurs public et privé, et les autorités politiques cherchent actuellement à en étendre le champ d'application jusqu'à couvrir l'ensemble de la population. Cependant, la généralisation de ce système se heurte à un certain nombre de difficultés. Sa gestion est confiée à un Organisme public d'assurance maladie (OPAM) dépendant du ministère de la Santé, qui a la charge de développer ses propres structures de soins. Or, la répartition des bénéficiaires comme celle des équipements connaissent de fortes inégalités régionales : Le Caire et surtout Alexandrie, ville pilote pour l'application de l'assurance maladie, regroupent plus du tiers des bénéficiaires et des équipements alors que certaines régions sont totalement dépourvues de structures de soins attachées à l'OPAM ${ }^{29}$. En raison de la faiblesse des salaires, notamment dans la fonction publique, les cotisations dégagées restent relativement modestes, donc insuffisantes pour développer les équipements de façon adéquate. réalité égyptienne. Historiquement constitué pour assurer les catégories salariées, 
l'OPAM retient comme critères principaux l'activité ou la non-activité des personnes ainsi que leur revenu salarial. Or la réalité socio-économique est beaucoup plus complexe. En effet, l'activité féminine est largement sous-évaluée; les enfants, qui représenteraient $10 \%$ de la population active, travaillent de façon illégale et les Égyptiens, surtout les fonctionnaires, cumulent fréquemment plusieurs emplois qui échappent au calcul des cotisations. Par ailleurs, le calcul sur la base d'un salaire fixe exclut les travailleurs temporaires, qui vivent dans des conditions très précaires, et ceux du secteur informel, pourtant de plus en plus nombreux. L'OPAM se heurte donc à de sérieux obstacles pour atteindre les catégories les plus défavorisées, qui pâtissent en outre des difficultés rencontrées par l'État pour proposer gratuitement un service médical de qualité, et qui risquent de se trouver bientôt exclues de toute protection et recours contre la maladie.

Enfin, le développement du système d'assurance maladie est handicapé par son assimilation au secteur public. En effet, les remboursements par les caisses de l'OPAM ne s'étendent qu'aux actes effectués directement dans les structures publiques, ou bien les propres établissements de soins de l'OPAM proposent des prestations gratuites pour les bénéficiaires. En reproduisant les modes de fonctionnement du secteur public de santé, il en emprunte également les blocages et les lourdeurs. Le fonctionnement bureaucratique d'une telle administration, le manque de moyens financiers qui restreint la qualité des équipements et des prestations ont conduit à un discrédit de ce système équivalent à celui dont souffre le secteur public de santé. De nombreuses personnes, affiliées dans le cadre de leur emploi, ont en fait recours, en cas de problèmes de santé, à d'autres formules de soins, notamment le secteur privé non remboursé. En demeurant dans le cadre du secteur public, le système d'assurance maladie ne contribue en rien au développement d'une médecine libérale.

Malgré les ambitions affichées, l'État ne parvient donc pas à étendre son offre de soins et à améliorer significativement leur qualité. Par ailleurs, il n'a pas su éviter l'accélération de la tendance vers une médecine à deux vitesses qui voit le médiocre système public de santé cohabiter avec un secteur privé de pointe réservé aux seules catégories supérieures. Dans ces conditions, l'État se trouve rapidement concurrencé dans son propre rôle, et l'islam se présente désormais, à son tour, en régulateur de la redistribution et en garant de la morale sociale et professionnelle.

\section{La médecine moderne au miroir de l'islam}

\section{Une mobilisation catégorielle au sein du Syndicat des médecins}

51 Depuis maintenant deux décennies, l'Égypte assiste à un "réveil de l'islam » dont l'idéologie, ou plutôt les idéologies, traversent l'ensemble des secteurs sociaux et économiques. La médecine ne fait pas exception et c'est au sein du Syndicat des médecins que l'offensive politique des courants se réclamant de l'islam a d'abord trouvé un terrain favorable. Les affrontements idéologiques entre défenseurs d'une médecine libérale et promoteurs d'une médecine socialisée, dont le syndicat a été le théâtre au cours de la période nassérienne, ont conduit à une quasi-paralysie de cette instance. L'élite traditionnelle en a été évincée et s'est repliée sur des positions académiques. À la fin des années soixante-dix, période au cours de laquelle se structure la mouvance islamiste, le syndicat des médecins, comme la plupart des autres syndicats professionnels, offre une sorte de «cadre vide» dans lequel cette mouvance trouve 
l'opportunité de se glisser et à travers lequel elle tente d'islamiser les pratiques sociales, élargissant ainsi son champ d'intervention à une sphère de plus en plus large.

Dès 1984, les militants islamistes obtiennent 7 des 25 sièges du Conseil national du syndicat des médecins, totalisant $39,5 \%$ des voix, et s'assurent également le contrôle d'importantes unions locales comme celles d'Alexandrie, du Caire et d'Assiout. Les élections de 1988 puis celles de 1992 confirment nettement cette percée puisque le courant islamiste emporte, dans les deux cas, plus de la moitié des sièges du Conseil national.

Sous l'impulsion de ce nouveau venu, le syndicat revient à ce qui devrait être une de ses fonctions principales : son rôle social, jusqu'alors quelque peu négligé. À travers de vastes programmes d'amélioration des conditions matérielles des médecins, le syndicat apparaît comme un laboratoire destiné à tester une mobilisation «catégorielle » de la société, ciblant son action sur les "jeunes médecins». La montée en puissance du courant islamiste semble en effet directement liée au rajeunissement de la profession ${ }^{30}$ et, en retour, il entreprend de traiter en priorité les problèmes spécifiques de cette catégorie. Ainsi, après les élections de 1984, un système d'assurance-maladie pour les médecins et leur famille a été mis en place, le syndicat participant pour les deux tiers aux dépenses de santé des praticiens cotisant à cette caisse. Par ailleurs, l'opération a été couronnée par l'ouverture au Caire d'un hôpital destiné aux seuls médecins ${ }^{31}$. Le Conseil élu au cours des élections suivantes a, quant à lui, entrepris l'élaboration d'un vaste projet d'amélioration des revenus des médecins, avec la volonté évidente de parvenir à une véritable autorégulation de la profession, dont la moralisation, de l'avis général, est liée à une revalorisation. Outre l'amélioration des salaires dans le secteur public, le projet redéfinit les fonctions qui y ont cours, en vue d'accroître la flexibilité dans l'exercice de la pratique et d'éviter le blocage actuel des promotions ${ }^{32}$. Par ailleurs, les instances du syndicat réclament l'augmentation de la part de la santé dans le budget national jusqu'à hauteur de $5 \%$, et cherchent à imposer un numerus clausus sévère pour limiter les inscriptions dans les facultés de médecine et à promouvoir des conditions d'études meilleures et surtout plus équitables entre les héritiers des mandarins et les étudiants issus des classes moyennes.

Par ailleurs, outre son rôle social, le syndicat tente de redonner au corps médical son rôle d'élite sociale, d'intermédiaire entre l'État et la société, qu'une certaine diminution de son prestige ainsi que sa subordination au projet étatique de médicalisation avaient quelque peu fait oublier. Les médecins sont ainsi invités à intervenir sur l'ensemble des problèmes de la société égyptienne et le syndicat, dans ce contexte, devient non seulement une institution de défense des intérêts professionnels mais également une tribune où peut librement s'exprimer ce " regroupement de gens instruits, fraction de la classe moyenne qui joue un rôle fondamental dans le système de relations entre les hautes et les basses classes de la société $\aleph^{33}$. Si cette vocation sociale réaffirmée a surtout donné lieu, au sein du syndicat, à des débats portant sur des sujets tels que la sédition confessionnelle, la torture, les méthodes de lutte contre le terrorisme ou encore le rôle de la jeunesse musulmane face aux défis du monde contemporain, elle est également parvenue à des réalisations concrètes; par exemple, à l'occasion du tremblement de terre d'octobre 1992, elle a réussi à mobiliser efficacement les membres du syndicat qui, grâce à une action médicale et, plus généralement, sociale, ont pu suppléer aux carences de l'État. En redonnant un pouvoir social aux médecins, le 
courant islamiste contribue à réconcilier ceux-ci avec la dimension philanthropique attachée à la pratique de leur profession ${ }^{34}$.

La référence islamiste est donc largement utilisée dans le but de réorganiser la profession sur des bases plus égalitaires et de réhabiliter un corps médical discrédité par son manque d'enthousiasme à participer à un processus de couverture sanitaire de base qui menace, il est vrai, une partie de ses intérêts professionnels. Par ailleurs, elle constitue une réelle nouveauté dans le champ médical en alliant désormais étroitement science et religion. Le malaise qu'elle révèle ne semblait pas toucher - ou du moins, à peine effleurer - les générations de la première moitié du $\mathrm{XX}^{\mathrm{e}}$ siècle qui manifestent, dans leur ensemble, une acculturation sereine. Le repli actuel sur le référent religieux est sans doute à mettre au compte de l'anomie résultant du double processus de massification et de déclassement de la profession. Cependant, il est également dû au fait qu'on ne peut plus avoir, aujourd'hui, une confiance aussi aveugle qu'auparavant en une science qui a montré ses limites - limites que ne lui soupçonnaient pas les générations précédentes. De plus, les récents progrès médicaux ont soulevé de nouveaux défis moraux provoquant, partout dans le monde, un retour de la philosophie et des religions dans le domaine des sciences. Aussi, au-delà du projet professionnel, l'islam entreprend-il de pénétrer le domaine de la science elle-même. Depuis une quinzaine d'années en effet, un certain nombre de scientifiques musulmans cherchent à définir les attributs d'une science, et donc d'une médecine, spécifiquement "islamique", en réponse à la science occidentale, réputée athée, dont les pays musulmans ont hérité à l'heure de son triomphe. Cependant, il s'agit moins, dans cette tentative, de proposer un modèle scientifique alternatif que de redonner un cadre éthique à une profession dont la morale s'est érodée, sans remettre en cause les principes essentiels de la biomédecine.

\section{Les cliniques islamiques : le libéralisme «à visage humain »}

Dans les années quatre-vingt, à l'heure même où émerge le concept de "médecine islamique ", le courant islamiste entreprend de déployer ses activités non plus dans la seule sphère politique mais également dans le domaine social. Cette nouvelle direction s'exprime notamment à travers les mosquées-centres sociaux, à la fois lieux de culte, crèche, école et clinique. L'Égypte est le premier pays à avoir assisté à ce phénomène, désormais présent également en Jordanie et en Algérie. Cette expérience n'est cependant pas entièrement nouvelle : la prise en charge des problèmes de santé par les associations religieuses et communautaires est traditionnelle en Égypte, et les églises chrétiennes possèdent, elles aussi, un important réseau de cliniques et de dispensaires.

Sans avoir connu l'extension qu'on a parfois voulu lui accorder, le phénomène des associations islamiques a connu une forte progression depuis les années soixante-dix ${ }^{35}$. Toutes les sensibilités d'un courant islamiste à multiples facettes s'y trouvent représentées. Ainsi, la très officielle banque Nâsir al-ljtimâ'î, qui collecte une part importante de la zakât (aumône légale), finance grâce à celle-ci de nombreux dispensaires de mosquées. Mais les réseaux non officiels de zakât, qui drainent, semble$\mathrm{t}$-il, des sommes fort importantes, opèrent des réalisations semblables auprès de mosquées appartenant à des courants fondamentalistes. Quant aux monarchies pétrolières du Golfe, elles ont sans doute une part importante dans le financement de certaines de ces mosquées-centres sociaux. 

l'offre de prestations médicales dont les revenus contribuent à financer les autres activités. Les cliniques de mosquées sont surtout installées en zones urbaines, notamment dans les villes de Moyenne-Égypte ${ }^{36}$, le plus souvent dans les quartiers populaires où les structures sanitaires étatiques sont absentes, et les honoraires des médecins libéraux trop élevés pour la population. Ces établissements ont généralement un niveau d'équipement satisfaisant et dispensent des soins variés et de qualité, pour un prix accessible, aux catégories défavorisées et aux classes moyennes déçues des prestations du secteur public de santé. Si les services proposés emportent la confiance de patients qui estiment le service public déshumanisé, et s'ils peuvent revendiquer un aspect «social» par opposition à l'élitisme du secteur privé, ils ne sont en rien l'expression d'un renouveau de la tradition médicale classique, contrairement à ce que l'on a pu observer ailleurs, notamment en Inde ${ }^{37}$. Dans un contexte de désengagement de l'État, ils apparaissent plutôt comme un recours offert au plus grand nombre pour bénéficier, dans de bonnes conditions, de la biomédecine occidentale en évitant les excès du libéralisme. Elles offrent ainsi une pratique alternative. Leur réussite tient sans doute également au fait que la référence religieuse contribue à rassurer des patients confrontés à une médecine reposant sur des principes fort différents de leurs propres représentations du corps et de la maladie. Dans une société où la médicalisation reste fragile, les malades risquent de chercher refuge ailleurs que dans le savoir médical. En témoigne, par exemple, le succès récent des traitements par le Coran. Certains cheikhs ont ainsi bâti leur réputation en soignant les maux du corps et de l'esprit par l'écoute de cassettes du Livre saint, que le patient accompagne de prières et de répétitions du nom de Dieu. En médiatisant ce besoin rassurant de religion, les dispensaires de mosquée tentent de réconcilier les représentations traditionnelles de la maladie et la médecine allopathique.

énomène des cliniques islamiques cherche donc à s'imposer comme une option concurrente face aux déficiences de l'État et aux excès du libéralisme. Les acteurs qui tentent de le promouvoir ne sont toutefois pas tous des islamistes radicaux. Les cliniques fondées par les Gamâ'ât islâmiyya, associations radicales, côtoient des dispensaires où le référent musulman témoigne surtout d'une tradition de bienfaisance. Au centre, l'État égyptien, qui se réclame également de l'islam, tolère ce type d'établissement puisqu'il y voit une réponse à sa difficulté à assumer sa vocation sociale. Cependant, les cliniques islamiques subissent également un contrôle visant à prévenir une mobilisation conduite par les mouvements islamistes ${ }^{38}$. Quoiqu'il en soit, elles ne s'opposent en rien aux orientations modernistes de l'État et contribuent même largement à la consommation de technologie médicale occidentale. En somme, loin de représenter une tentative de fusion des approches médicales traditionnelle et moderne, les cliniques islamiques cherchent à se réapproprier la biomédecine occidentale sur un modèle familier, acceptable pour la population, tout en constituant une importante opportunité d'emploi pour les jeunes médecins. Elles participent par ailleurs d'un dispositif plus général de recomposition des modes de pratique, qui se déclinent autour du modèle libéral, en allant du gigantesque hôpital privé au modeste cabinet de clientèle, dans la diversité desquels chaque médecin tente de trouver la formule qui lui permettra de parvenir à une certaine réussite sociale tout en participant à l'élaboration d'un nouveau système de santé visant à s'ajuster à toutes les catégories de la société égyptienne. 


\section{La délicate ouverture du marché médical}

60 La loi $\mathrm{n}^{\circ} 43$ de 1974 sur les investissements arabes et étrangers a permis une offensive du secteur privé dans le domaine de la santé jusqu'alors très largement dominé par les structures publiques de soins. Cette privatisation a consisté, dans un premier temps du moins, à la création de grands hôpitaux privés, dits "d'investissement ", équipés d'instruments de haute technologie et répondant aux normes les plus modernes des établissements de santé. La médicalisation rapide du pays, en créant de nouveaux besoins, surtout parmi les catégories aisées, semblait en effet permettre l'implantation de ce type d'unités, aptes à offrir les services des spécialités médicales les plus pointues, mais que l'État n'était pas toujours en mesure de prendre en charge. L'ouverture au secteur privé visait ainsi à provoquer la création de tels établissements, de niveau comparable à ceux des pays occidentaux, afin de limiter les séjours curatifs à l'étranger, d'attirer la clientèle arabe du Golfe et de tenter de faire revenir au pays les spécialistes égyptiens pratiquant hors du territoire.

61 Ces hôpitaux d'investissement, dont le nombre a augmenté ces dernières années, recouvrent des réalités assez différentes. Après les gigantesques usines médicales fondées dès le lancement de la politique d'ouverture économique, ce sont aujourd'hui des structures plus modestes qui sont privilégiées. Tous ces établissements partagent cependant une conception très commerciale de la médecine : la moindre prestation $\mathrm{y}$ est payante, y compris les seringues et le coton utilisés pour les injections, ou encore le plâtre et les bandages. Les tarifs pratiqués, bien que soumis à de fortes variations d'une clinique à une autre, sont toujours élevés. Seule une minorité de patients peut avoir accès aux principaux établissements mais, grâce à la multiplication récente de structures plus réduites, ces cliniques drainent désormais également une population issue des classes moyennes, voire modestes, lassées des insuffisances du secteur public.

62 Ce succès grandissant est cependant pondéré par la réputation douteuse de certains établissements. Les hôpitaux d'investissement sont en effet au centre de scandales retentissants et répétitifs. Si ces scandales, au départ, concernaient surtout leurs modes de financement et l'enrichissement jugé indécent de leurs dirigeants, c'est aujourd'hui autour des erreurs médicales, des négligences professionnelles et des débordements éthiques que s'expriment les principaux griefs.

63 Ces établissements n'ont certes pas le monopole des erreurs médicales, qui semblent de plus en plus fréquentes puisque l'ensemble du corps médical est régulièrement mis en cause, notamment dans la presse. Cependant, un certain nombre de négligences aux conséquences dramatiques ont récemment attiré l'attention du public et des autorités. Ainsi, en 1993, les centres de dialyse se sont trouvés au cœur d'une affaire de contamination par le virus du sida. Les premiers accusés sont les laboratoires biologiques privés, auxquels les centres de dialyse font appel pour réaliser les analyses de sang préalables aux opérations de dialyse. Certains résultats se sont révélés erronés, masquant là séropositivité de quelques patients. Il est vrai qu'à l'occasion, la responsabilité du secteur public, auquel sont attachés ces centres de dialyse, est également en cause puisque la réutilisation des appareils et des filtres pour plusieurs patients, sans qu'il ait été procédé aux mesures normales de désinfection, a contribué à l'extension du virus à une douzaine de personnes. Dans ces circonstances, c'est cependant aux laboratoires privés qu'on a fait porter l'essentiel de la responsabilité de cette affaire. L'État a donc interdit le recours aux établissements privés pour effectuer 
les analyses sanguines des patients dont l'état de santé nécessite une dialyse; désormais, seuls les laboratoires dépendants du ministère de la Santé sont habilités à réaliser ces examens. À cela s'ajoutent des consignes de ce même ministère pour inciter à utiliser plus scrupuleusement les appareils de dialyse.

Ainsi, malgré la volonté affichée de poursuivre dans ce sens, la privatisation du système de santé ne laisse pas d'inquiéter les autorités comme les utilisateurs. Dans un secteur encore très peu réglementé, les dérives sont toujours possibles et le spectre d'une médecine "commerciale " se profile à l'horizon de cette ouverture au marché du secteur médical. Dans une logique où tout est à vendre, l'éthique médicale se trouve vite menacée. On a vu récemment surgir une nouvelle affaire révélant un trafic de faux certificats octroyés par les laboratoires biologiques de certains hôpitaux d'investissement, déclarant faussement indemnes du virus de l'hépatite $\mathrm{C}$ les candidats à l'émigration vers les pays du Golfe qui pouvaient en payer le prix. En effet, certains pays d'accueil refusent d'admettre les individus dont les analyses sont positives; or, une part très importante de la population égyptienne est porteuse de ce virus.

C'est cependant le trafic d'organes qui constitue l'élément le plus inquiétant dans le contexte actuel. Les déficiences rénales sont très fréquentes en Egypte et le traitement par dialyse particulièrement coûteux. La greffe se révèle à terme moins onéreuse et représente souvent l'unique espoir du patient. L'importance du marché a finalement donné naissance à un véritable trafic de reins, dont les hôpitaux d'investissement semblent constituer le pivot, et les « vols » d'organes sur des patients sains ne sont pas rares dans ce type d'établissements. La médecine des hôpitaux privés ne doit bien sûr pas être réduite à ces cas extrêmes et elle compte par ailleurs des praticiens de valeur, mais les scandales qui l'agitent révèlent de façon très visible les dérives auxquelles elle peut donner lieu. Face aux dangers présents et à venir, l'État tente, souvent en vain, notamment en mobilisant sans grand succès un dispositif législatif encore embryonnaire, de garder un certain contrôle sur le processus en cours.

À côté de la vitrine technologique que constituent les hôpitaux d'investissement, la privatisation du système de santé a pris la forme de modestes cabinets de consultation qui se sont rapidement multipliés ces dernières années. En raison des représentations dominantes de la profession, d'une part, et du discrédit porté sur le secteur public, d'autre part, l'acquisition d'un cabinet de clientèle reste pour les médecins le but ultime, la consécration d'une certaine réussite. On estime qu'il faut environ 10000 livres égyptiennes pour équiper un cabinet médical, mais beaucoup de jeunes médecins se lancent dans l'aventure avec infiniment moins de moyens. On trouve ainsi un grand nombre de ces cabinets dans les quartiers populaires, installés dans des logements minuscules et sans confort et équipés du strict minimum. La démarche de ces praticiens emprunte à la logique de ce que l'on désigne en Égypte par le terme d' « informel », qui s'applique traditionnellement à l'habitat ou au petit commerce, par exemple. Dans le secteur médical, les médecins s'improvisent aussi petits entrepreneurs et tentent leur chance au sein d'une structure modeste, établie souvent à la limite de la légalité. Leurs propriétaires, en effet, n'ont pas toujours le droit d'exercer dans le privé ${ }^{39}$, ou bien ne déclarent pas leur cabinet pour échapper l'impôt.

Si beaucoup de ces cabinets sont d'un rapport très médiocre, les médecins y trouvent cependant une gratification symbolique à travers laquelle ils commencent à se réconcilier avec l'image de soi. Par ailleurs, la multiplication de ces cabinets a permis une répartition plus équilibrée des services de santé, en particulier dans les zones 
urbaines défavorisées, dépourvues de structures publiques de soins, et même, désormais, en milieu rural. Cependant, la qualité des prestations y est très inégale et souvent fort médiocre. La baisse du niveau d'enseignement menace en effet la compétence des praticiens, qui arrivent sur le marché médical avec des connaissances lacunaires et sans aucune formation pratique. Là encore, la fréquence des erreurs médicales alerte l'opinion et menace de discréditer la profession.

En outre, dans un contexte de forte concurrence, de nombreux praticiens privés recourent à une stratégie de distinction qui repose sur une supercherie, afin d'échapper à la dévalorisation du statut de généraliste : elle consiste à abuser du titre de spécialiste quand on ne l'est... que sur la plaque professionnelle placée à la porte du cabinet. Il y aurait plusieurs centaines de contrevenants pour la seule ville du Caire. Or, l'administration du ministère de la Santé ne compte qu'un seul inspecteur par gouvernorat pour contrôler ce type d'infractions. L'ouverture du secteur médical à la loi du marché appellerait pourtant, et de toute urgence, une réglementation ou, du moins, des modes de régulation visant à sauvegarder l'éthique professionnelle.

Dans le domaine de la santé, la privatisation entrâne donc une série de problèmes où la morale est la première mise en danger, ainsi que la qualité des prestations et l'égalité d'accès aux soins. Les médecins ne sont ni ignorants ni insensibles à ces problèmes et certains d'entre eux, certes très marginalement, commencent à se mobiliser dans le cadre du mouvement associatif. Cependant, ils se découvrent particulièrement démunis face à l'ampleur de la tâche à accomplir. Aussi recherchent-ils souvent des compétences et une collaboration efficace auprès des organismes internationaux ou des $\mathrm{ONG}^{40}$, envers lesquelles les médecins égyptiens éprouvent dans leur ensemble un véritable engouement. Pendant longtemps en effet, l'État a offert un modèle en quelque sorte "préfabriqué ", réputé juste et équitable, sur lequel les praticiens n'avaient qu'à se reposer en confiance, sans plus avoir à penser eux-mêmes au sens de leur vocation sociale. Aujourd'hui, alors que ce modèle a fait la preuve de son incapacité à résoudre tous les problèmes, il revient aux médecins d'inventer et de mettre en œuvre un nouveau modèle où la justice et l'équité restent d'autant plus d'actualité que le contexte est à la démocratisation. Mais alors que leur participation à la réflexion est encouragée, les médecins se révèlent incapables, faute d'une formation adéquate et d'un niveau de culture générale et de conscience suffisant, de mener à bien cette réflexion. L'enseignement de la médecine, très technique, ne donne pratiquement aucune place aux sciences sociales et le système, basé sur l'obsession de la réussite aux examens, ne laisse aucun loisir pour se pencher sur la compréhension du monde. Dans un contexte où la mobilisation politique, qui offrait des outils pour guider la réflexion, est largement retombée, ce sont bien souvent les stratégies individuelles ouvrant la porte aux débordements éthiques qui guident finalement l'action des médecins.

71 C'est précisément l'alliance de l'incapacité des médecins à gérer eux-mêmes les problèmes posés par l'évolution du système de santé, d'une part, et des risques de dérives morales du système - danger qui ne leur échappe pas -, d'autre part, qui donne au modèle islamique son caractère opérationnel. En effet, celui-ci se veut à la fois mode d'exercice professionnel et garantie de moralité. En ce sens, la capacité théorique de contrôle du syndicat sur la pratique, à travers le rôle du comité de déontologie (lagna adâb), auquel est associé un conseil de discipline, est un enjeu symbolique, sinon efficace, de la mainmise des islamistes sur le syndicat. Ceux-ci possèdent ainsi un outil 
grâce auquel ils peuvent concurrencer l'État dans un rôle de régulation du processus de privatisation et de sauvegarde de la morale professionnelle et ils sont en mesure, par ailleurs, de proposer un modèle médical qui se veut adapté à la situation sociale égyptienne, celui des dispensaires de mosquée, qui a fait preuve jusqu'à présent de son efficacité.

Dans le domaine de la santé, la libéralisation économique s'avère délicate. D'une part, l'État ne peut se permettre de livrer ce secteur aux seuls capitaux privés, ce qui exclurait automatiquement de larges portions de la population de tout accès aux soins. D'autre part, la situation économique fragile et les difficultés, dans le contexte égyptien, à généraliser un système d'assurance-maladie ne permettent pas à l'État de diversifier lui-même le système médical et d'offrir ainsi des opportunités d'emploi aux médecins. Finalement, ceux-ci se mobilisent individuellement, ou collectivement dans le cadre de leur syndicat, pour forger des espaces de pratique dont, bien souvent, la référence à l'islam est supposée garantir la moralité. Il est encore difficile de mesurer l'impact sur la santé de la population d'un processus entamé à la fin des années quatrevingt. Mais si la libéralisation en cours du système de santé est parvenue, malgré son caractère quelque peu anarchique, à diversifier et à étendre géographiquement les lieux d'accès aux soins, elle doit désormais s'accompagner d'une réglementation cohérente, capable de contenir les débordements éthiques.

\section{NOTES}

1. À l'instigation de Muhammad 'Ali, une École de médecine est fondée au Caire en 1827 par un médecin français, Antoine-Barthélémy Clot, où l'on enseigne les principes de la médecine moderne alors en plein renouvellement.

2. Fondé également par Clot en 1840, le service civil de santé, destiné prioritairement aux zones rurales, employait, avec un statut de fonctionnaire, les médecins formés à l'École de médecine du Caire. Cependant, c'est au travers de l'exercice libéral, souvent cumulé avec un emploi dans le service public, que les médecins égyptiens ont acquis leur position sociale et accédé à la notabilité.

3. MAHFOUZ N., Le Palais du désir (trad. Ph. Vigreux), J. C. Lattès, Paris, 1987, p. 75.

4. BINDER L, In a Moment of Enthusiasm, Political Power and the Second Stratum in Egypt, University of Chicago Press, Chicago \& Londres, 1978.

5. MOORE C. H., Images of Development. Egyptian Engineers in Search of Industry, MIT Press, Cambridge, 1980, p. 45.

6. BUTTER I. \& MEJIA A., « Trop de médecins ! ", Forum mondial de la santé, vol. 8,1987, p. 542.

7. ARLIAUD M., Les médecins, La Découverte, Paris, 1987.

8. MOULIN A.-M., "La profession médicale dans les pays arabes : vues historiques à long et à court termes ", Médecins et protection sociale dans le monde arabe, Cahiers du Cermoc n 5 , Amman, 1993, p. 228.

9. ARLIAUD M., op. cit., p. 104.

10. MORSY S. A., "Islamic Clinics in Egypt: The Cultural Elaboration of Biomedical Hegemony », Medical Anthropology Quarterly, vol. II, n 4, décembre 1988, p. 264. 
11. La médecine ne détient pas le monopole des critiques concernant le niveau d'enseignement, et le système universitaire fait l'objet de multiples débats dans la société égyptienne.

12. "Proclamation of the National Charter", President Nasser's Speeches and Interviews, Information Department, Cairo, 1962, p. 335.

13. Jusqu'alors, c'est le Journal de l'Association médicale égyptienne, fondé en 1917, qui était l'organe d'expression majeur des médecins, du point de vue scientifique comme du point de vue professionnel.

14. Unité urbaine de base du système de santé. Son rôle principal est d'enregistrer les naissances et décès et de pratiquer la vaccination.

15. IDRIS Y., "Vieillesse sans démence », Maison de chair, Sindbad, Paris, 1989, p. 148-166.

16. AL-GAWÂD Y. M., Al-siha wa-l-tibb wa-l-'ilâj fi Misr (la santé, la médecine et les soins en Égypte), Université de Zagazig, 1987.

17. A Report on Health Development in the ARE. À Sector in Transition, US AID, mai-juin 1982.

18. AL-GAWÂD Y. M., op. cit., p. 8.

19. Ibid., p. 12.

20. Le cumul des modes de pratique publique et privée est cependant de plus en plus fréquent; cf. infra.

21. Il s'agit du salaire de base, auquel il convient d'ajouter les primes qui peuvent représenter jusqu'à $50 \%$ du montant du salaire.

22. Al-Sha'b, 29/07/86.

23. Al-Atibbâ' n 104 , janvier 1989, p. 49.

24. L'analyse du sondage ne donne malheureusement pas le nombre des médecins qui ont répondu au questionnaire. Al-Atibbâ' n 104, janvier 1989, p. 18-19.

25. UNICEF, La situation des enfants dans le monde, 1989, p. 94.

26. Ce projet de convention, finalement adopté le 20 novembre 1989, a donné lieu à une Conférence nationale à Alexandrie au cours de laquelle ont été discutés certains points du projet, contestés par les pays du tiers-monde. Mme Moubarak, qui présidait cette Conférence, a reçu en 1989 le prix Maurice Beth (ce prix est décerné par l'Unicef à une personnalité ou une institution ayant eu une action particulièrement efficace dans le domaine de l'enfance).

27. World Development Report 1993. Investing in Health, World Bank, Oxford University Press, 1993, p. 294.

28. Les projets d'offre de soins à la paysannerie ont, quant à eux, toujours reposé sur le principe de la gratuité pour l'usager, en raison de la très faible monétarisation de cette catégorie. Ce n'est que depuis ces dernières années que l'on commence à envisager un système d'assurance maladie pour les paysans.

29. Taqrîr 'an al-ta'mîn al-sihî al-ijtimâ'î (Rapport sur l'assurance sociale maladie), mai 1985, p. 7-8. 30. De 1984 à 1988, 7000 nouveaux médecins s'inscrivent sur le registre du syndicat et l'on compte 7000 voix de plus pour la liste islamique aux élections de 1988, par rapport à celles de 1984.

31. Al-Atibbâ' n' 100 , mars 1987, p. 6-7.

32. Al-Atibbấ' n' 103 , octobre 1988, p. 31-40.

33. Al-Atibbâ' $n^{\circ} 101$, novembre 1987, p. 20.

34. Cependant, la référence à l'islam peut conduire également à des prises de position moins généreuses, voire dangereuses : la tentation d'exclure certaines catégories de malades, victimes du sida ou de l'alcoolisme, par exemple, en demeure sans doute la manifestation la plus inquiétante, bien qu'encore marginale.

35. BEN-NEFISSA S., «Le mouvement associatif égyptien et l'islam. Eléments d'une problématique ", Maghreb-Machrek n 135, janvier-mars 1992, p. 19-36.

36. BEN-NEFISSA S., art. cit. p. 27. 
37. ZIMMERMANN F., Le discours des remèdes au pays des épices. Enquête sur la médecine hindoue. Paris, Payot, 1989. En Inde, les érudits ont perpétué jusqu'à nos jours la tradition ayurv2dique et se sont dotés d'institutions propres. L'idéologie de la douceur contenue dans cette médecine a pu ainsi s'affirmer contre le système allopathique importé par les Occidentaux.

38. FARAG I., "Croyance et intérêts: réflexion sur deux associations islamiques », in Modernisation et nouvelles formes de mobilisation sociale, Égypte-Turquie, Cedej, Le Caire, 1992.

39. Bien que le cumul des modes d'exercice soit de nouveau autorisé et très largement pratiqué, certains postes de la fonction publique (responsable d'un bureau de santé, inspecteur provincial...) n'autorisent pas, en principe, un tel cumul.

40. Ces institutions offrent en effet le visage rassurant de la compétence grâce à l'expertise occidentale et aux atouts de la technologie, tout en conservant une vocation sociale, voire humanitaire, qui séduit les médecins égyptiens, sensibles aux immenses lacunes médicales de leur société.

INDEX

Mots-clés : médecine, santé

\section{AUTEUR}

SYLVIA CHIFFOLEAU

CNRS 